\title{
Modelo de negocio para optimizar las estrategias productivas de personalización en masa
}

Luis Miguel Arroyo Gutiérrez, Montserrat Jiménez Partearroyo, Carmen de Pablos Heredero Universidad Rey Juan Carlos (Spain)

luismiguel.arroyo@urjc.es, montserrat.jimenez@urjc.es, carmen.depablos@urjc.es

\section{Resumen}

Objeto: Describir un modelo general de proceso y negocio de personalización en masa. Destacar los elementos claves para llevar a cabo la personalización en masa como estrategia productiva principal o complementaria de una empresa. El modelo propuesto se basa en una revisión exhaustiva de la literatura y se define en su completa dimensión, describiendo diferentes etapas, elementos, interrelaciones y flujos de información; ofreciendo una perspectiva global hasta ahora inexistente en la literatura.

Diseño/metodología/enfoque: Se ha llevado una exhaustiva revisión de la literatura y no se ha encontrado un modelo o estructura del proceso de personalización en masa universalmente aceptada, ni tampoco una estructura amplia y de conjunto, que cubra y describa el proceso completo y pueda asimilarse a un modelo de negocio. Sin embargo en la literatura se ofrecen cinco enfoques parciales y diferentes en las que los autores se han basado para proponer un modelo de negocio integrado de personalización.

Aportaciones $\boldsymbol{y}$ resultados: En este trabajo se ha definido y desarrollado el proceso general de implantación de la personalización en masa en tres etapas, poniendo énfasis en las iteraciones que existen entre las ellas que le convierten en proceso retroalimentado. Se ha encontrado que el modelo resulta especialmente útil en la generación de valor. El modelo expone los principios para identificar el valor y como la generación de valor refleja la particularidad de la personalización en masa frente a otras estrategias. 
Limitaciones: El modelo de negocio presentado es teórico, por ello es interesante desarrollar un contraste empírico. Así mismo, al ser un modelo de aplicación general no ha sido posible entrar en las peculiaridades de cada industria.

Implicaciones prácticas: La estrategia productiva de personalización en masa permite la generación de valor ofreciendo productos que satisfacen preferencias individuales, con costes y eficiencias similares a los de producción en masa, por tanto integra las ventajas de las estrategias competitivas de diferenciación y bajo coste. La definición del modelo de negocio propuesto permite a los directivos reflexionar sobre como implementar esta estrategia y como valorar las características personalizables de los atributos del producto a ofertar, para generar valor.

Implicaciones sociales: Dentro de la estructura competitiva del mercado global actual, la estrategia de personalización en masa se puede contemplar como una fórmula innovadora para poder posicionarse competitivamente, que además se acepta bien por los consumidores. Por tanto, es necesario definir claramente un modelo de negocio asociado a dicha estrategia.

Originalidad / Valor añadido: En este artículo se propone un modelo de negocio para la estrategia productiva de personalización en masa, que contempla todas las etapas, sus interrelaciones y flujos de información que se originan en todas sus dimensiones. Un modelo completo que no se puede encontrar en la literatura y que puede facilitar el conocimiento y entendimiento de la personalización en masa por parte de la industria y sus directores funcionales y generales, comunidad en la que hasta este momento la personalización en masa ha tenido muy poca aceptación y difusión real.

Palabras clave: Personalización en masa, Estrategia productiva, Diferenciación, Estandarización personalizada

Códigos JEL: L6, L1

Title: Mass customization business model for the optimization and implementation of the strategy

\section{Abstract}

Purpose: Describe a general process and business model for mass customization, highlighting the key elements for its implementation, either as a central or complementary production strategy. The proposed model is based on a thorough literature review and is defined in its full dimension, describing the different stages, 
elements, inter-relationships and information flows; offering a global perspective that could not be found in the existing literature.

Design/methodology: In the in-depth literature review performed, it was not possible to find a business model or structure for the mass customization process that was either generally accepted, or viewed the process with a broad perspective and as a whole. However, the literature offered five partial and different approaches, from which it was possible to build the proposed integrated business model for mass customization.

Findings: This work defines and develops an overall mass customization process, implementation and business model. The model has three stages and emphasizes the iterations that exist between them, including the feedback loop processes. The model is particularly useful to focus in the value creation. It sets out the principles for identifying value in the mass customization and how the value is generated in this particular strategy.

Research limitations/implications: The business model presented is theoretical, so it would be interesting to develop an empirical contrast. Likewise, been a model for general application, it has not been possible to enter into the peculiarities of each industry.

Practical implications: Mass customization productive strategy generates value by offering products that satisfy individual preferences, with costs and efficiencies similar to the mass produced ones. Therefore integrates the advantages of differentiation and cost leadership competitive strategies. The definition of the proposed business model can allow managers to reflect on how to implement this strategy and how to assess the value creation of the product customizable attributes.

Social implications: Within the current global market competitive structure, mass customization strategy can be seen as an innovative formula to enable the achievement of a competitive position well accepted by consumers. Therefore, it is necessary to count with a clear business model associated with this strategy.

Originality/value: This paper proposes a business model for mass customization strategy, which includes all stages, their interrelationships and consequent information flows. A complete model that cannot be found in the literature and that can provide the knowledge and understanding about mass customization. It may be of interest for the industry, especially for general and functional managers, a community that until this moment, mass customization has had little acceptance and actual diffusion. 
Keywords: Mass customization, Productive strategy, Differentiation, Customized standardization

Jel Codes: L6, L1

\section{Introducción}

El enfoque de la gestión de la producción tradicional que ha sido el paradigma industrial del pasado siglo, se basa en maximizar el beneficio disminuyendo los costes mediante la producción de productos estándar en altos volúmenes. Estos volúmenes permiten obtener importantes economías de escala, así como la especialización y mecanización de procesos, orientándose por tanto las empresas hacia la producción en masa. Esta estrategia supone dirigir las competencias hacia el liderazgo en costes, declinando diferenciar los productos y atender a una demanda hoy en día cada vez más exigente y global. Es por ello que las empresas deberían evolucionar desde un enfoque industrial endogámico basado en la eficiencia interna del proceso de producción, a un paradigma post-industrial orientado a proporcionar mayor valor añadido a los clientes, simultaneando sistemas de producción de grandes volúmenes y muy eficientes en coste, pero a su vez, personalizados a las necesidades de cada cliente.

De esta manera surge el concepto de "personalización en masa" (Davis, 1987) como una estrategia emergente competitiva y productiva, que según numerosos autores (Pine, 1999; Da Silveira, Borenstein \& Fogliatto, 2001; Fiore, Lee \& Kunz, 2004; Franke \& Schreier, 2008; Lee \& Chang, 2011; Zhong, Dai, Qu, Hu \& Huang, 2013; Piller \& Blazek, 2014), tendrá un crecimiento espectacular en los próximos años, ya que satisface una demanda creciente del mercado, difícilmente abordable por modelos tradicionales de negocio. Por tanto, promete ser, sin duda, una de las tendencias productivas más relevantes del siglo XXI. Sin embargo, su aceptación en el mundo empresarial es todavía muy escasa (Satam, Liu \& Lee, 2011; Bohnstedt, 2014; Moon \& Lee, 2014), habiendo contribuido a ello sin duda la complejidad aparente y la ausencia de un modelo de negocio generalmente aceptado que lo defina.

En el contexto de esta investigación se estima necesario contar con un modelo general de proceso y negocio de personalización en masa, por un triple motivo, el primero es que la definición de un proceso o modelo global ofrecerá la perspectiva necesaria para entender la personalización en masa en su completa dimensión, sus diferentes etapas, elementos, interrelaciones y flujos de información. La segunda es que un modelo puede facilitar el conocimiento y entendimiento de la personalización en masa por parte de la industria, sus directores funcionales y generales, comunidad en la que hasta este momento la 
personalización en masa ha tenido muy poca aceptación y difusión real. En tercer lugar esta estructura nos facilitará la definición de los elementos claves de la personalización en masa

El objetivo de este artículo es establecer de una forma reflexionada una propuesta de modelo de negocio que describa la personalización en masa de manera que, además de cubrir este vacío en la literatura, estimule a que las organizaciones implementen esta estrategia. Para ello este trabajo se estructura en los siguientes apartados. En el apartado dos se propone una definición de la personalización en masa, en el apartado tres se lleva a cabo una revisión de la literatura sobre las aportaciones que se han considerado más relevantes, en el apartado cuatro se realizará una propuesta de modelo de negocio de personalización en masa y en el último apartado, el cinco, se realizará una discusión del modelo y formularan las conclusiones.

\section{Definición de personalización en masa}

En la literatura no se ha encontrado una única definición consensuada del concepto que sea generalmente aceptada, por lo que se han examinado y recopilado las definiciones que sobre este concepto se han realizado por los investigadores (Tabla 1).

\begin{tabular}{|c|c|}
\hline Autor & Definición \\
\hline Davis (1987) & $\begin{array}{l}\text { La habilidad de entregar productos que satisfagan las necesidades individuales de } \\
\text { los clientes de forma rentable }\end{array}$ \\
\hline Pine (1993) & $\begin{array}{l}\text { Suministrar una ingente variedad y personalización individual, a precios } \\
\text { comparables a servicios y bienes de producción en masa }\end{array}$ \\
\hline Hart (1995) & $\begin{array}{l}\text { El uso de de procesos y estructuras organizativas flexibles para producir bienes y } \\
\text { servicios personalizados variados y a veces individuales al coste de productos } \\
\text { estándar producidos en masa }\end{array}$ \\
\hline $\begin{array}{l}\text { Ahlstromp y } \\
\text { Westbrook (199 }\end{array}$ & $\begin{array}{l}\text { Desarrollar los procesos que pueden suministrar una amplia gama de variantes de } \\
\text { los productos seleccionados por los clientes en cada pedido con pequeños } \\
\text { incrementos de coste o tiempo de entrega }\end{array}$ \\
\hline Tseng y Jiao & $\begin{array}{l}\text { Las tecnologías y sistemas para suministrar bienes y servicios que satisfacen las } \\
\text { necesidades individuales de los clientes con eficiencias cercanas a la producción en } \\
\text { masa }\end{array}$ \\
\hline Piller (2005) & $\begin{array}{l}\text { Proceso de codiseño con el cliente de productos y servicios que satisface las } \\
\text { necesidades individuales de cada cliente referidos a ciertas características del } \\
\text { producto, Todas las soluciones se realizan dentro del espacio fijo de solución, } \\
\text { caracterizado por un proceso estable pero flexible y que da respuesta. Como } \\
\text { resultado, los costes asociados con la personalización permiten un nivel de precios } \\
\text { que no implican el cambio a un segmento más alto del mercado }\end{array}$ \\
\hline $\begin{array}{l}\text { Kumar, Gattoufi y } \\
\text { Reisman (2007) }\end{array}$ & $\begin{array}{l}\text { La estrategia empresarial que permite construir alta personalización en un } \\
\text { producto a precios asociados con producción en masa, tiene tres elementos a } \\
\text { través de los cuales esto se hace posible: diseño modular del producto, espacio } \\
\text { solución finito y co-diseño con el cliente }\end{array}$ \\
\hline
\end{tabular}

Tabla 1. Recopilación de definiciones 
Analizando todas ellas y reflexionando sobre los conceptos básicos que aparecen como por ejemplo: satisfacción de preferencias individuales del cliente, espacio solución, eficiencia, codiseño, procesos y tecnologías, proponemos la siguiente definición: Se entiende la personalización en masa como la estrategia competitiva y productiva que permite la generación de valor ofreciendo productos que satisfacen preferencias individuales, con costes y eficiencias similares a los de producción en masa. El diseño del producto individual se realiza mediante el co-diseño con el cliente, este producto se enmarca dentro del un espacio fijo solución, que delimita la oferta y define los procesos, las tecnologías y el sistema productivo".

Este planteamiento tan sencillo contradice la memoria colectiva empresarial; a nivel de estrategia competitiva es una estrategia híbrida considerada muy débil (Porter, 1980) y a nivel de estrategia productiva tradicionalmente se ha considerado incompatible simultanear la producción en masa y la personalización (Selladurai, 2004; Dean, Xue \& Tu, 2009). Sin embargo, hoy en día esto es posible gracias entre otros elementos, a la aparición de las nuevas tecnologías flexibles de fabricación y de información. Las primeras permiten la fabricación de pequeños lotes de productos variados de manera económica y las segundas, el acceso a un mercado global y la implicación del cliente directamente, en el diseño y personalización de su producto.

\section{Revisión de la literatura}

En la revisión de la literatura realizada no se ha encontrado un modelo o estructura del proceso de personalización en masa universalmente aceptada, ni tampoco una estructura amplia y de conjunto, que cubra y describa el proceso completo y pueda asimilarse a un modelo de negocio. Los autores se centran en partes del proceso (Grenci \& Watts, 2007; Richards \& Jones, 2008), en el análisis de los factores que explican el comportamiento de los consumidores (Damm, De Pablos Heredero \& Rodríguez Monroy, 2013), casos de organizaciones con prácticas que ofrecen buenos resultados (Gallego Gómez \& de Pablos Heredero, 2013), o bien en aspectos concretos de la personalización en masa (Wang, Zeng, Koehl \& Chen, 2014; Shimada, Ogata, Ota \& Hara, 2014) y cuando se enfocan en aspectos más generales, éstos no tienden a analizar la globalidad. Sin embargo en la literatura encontramos cinco aproximaciones a la personalización que podemos asimilar a modelos, sin llegar ninguna a ser completa. Estas cinco aproximaciones son:

- Metodología para desarrollar una arquitectura de producto para la personalización en masa (Jiao \& Tseng, 1999)

- Bucle de información de la personalización en masa (Reichwald, Piller \& Moslein, 2000) 
- Bucle de conocimiento de la empresa (Tseng \& Piller, 2003)

- Cuatro niveles en personalización en masa (Tseng \& Piller, 2003)

- Sistemas de empresas personalizadoras (Blecker, Friedich, Kaluza, Abdelkafi \& Kreuutler, 2005)

Cada una de estas cinco aproximaciones o modelos, no sólo se centran en un área distinta sino que también analizan el proceso desde una perspectiva diferente, por lo que estudiados todos en conjunto aportarán la visión más global posible, que debería cubrir la mayoría del proceso. Otro factor clave que facilita el análisis es que no hay contradicciones entre las cinco aproximaciones, y por tanto, son compatibles entre sí. Dos de las aproximaciones, el Bucle de información (Reichwald et al., 2000) y el Bucle de conocimiento (Tseng \& Piller, 2003), comparten autor, por lo que existe una influencia obvia entre ellos, pero hay matices que es interesante comentar.

\begin{tabular}{|c|c|c|c|c|}
\hline Autores & Proceso & Enfoque & Elementos & Resultado \\
\hline $\begin{array}{l}\text { Jiao y Tseng } \\
\text { (1999) }\end{array}$ & $\begin{array}{l}\text { Metodología para } \\
\text { desarrollar una } \\
\text { arquitectura de } \\
\text { producto }\end{array}$ & $\begin{array}{l}\text { Desarrollo de } \\
\text { producto }\end{array}$ & $\begin{array}{l}\text { 1. Vista funcional } \\
\text { 2. Vista técnica } \\
\text { 3. Vista física }\end{array}$ & $\begin{array}{l}\text { Plataforma } \\
\text { de producto }\end{array}$ \\
\hline $\begin{array}{l}\text { Reichwald et } \\
\text { al. (2000) }\end{array}$ & $\begin{array}{l}\text { Ciclo de } \\
\text { información }\end{array}$ & $\begin{array}{l}\text { Sistemas de } \\
\text { información }\end{array}$ & $\begin{array}{l}\text { 4. Escuchar a los clientes } \\
\text { 5. Configuración de los productos } \\
\text { por clientes actuales o nuevos } \\
\text { (Co-diseño) } \\
\text { 6. Planificación de Fabricación } \\
\text { 7. Producción } \\
\text { 8. Distribución y gestión de la } \\
\text { relación. }\end{array}$ & $\begin{array}{l}\text { Flujos de } \\
\text { información }\end{array}$ \\
\hline $\begin{array}{l}\text { Tseng y Piller } \\
\text { (2003) }\end{array}$ & $\begin{array}{l}\text { Ciclo de } \\
\text { conocimiento }\end{array}$ & $\begin{array}{l}\text { Sistemas de } \\
\text { conocimiento }\end{array}$ & $\begin{array}{l}\text { 9. Definición del espacio solución. } \\
\text { 10. Configuración de producto para } \\
\text { clientes existentes y } \\
\text { configuración del producto para } \\
\text { clientes nuevos. } \\
\text { 11.Fabricación y entrega. } \\
\text { 12. Distribución y gestión de la } \\
\text { relación (CRM). }\end{array}$ & $\begin{array}{l}\text { Flujos de } \\
\text { conocimiento }\end{array}$ \\
\hline $\begin{array}{l}\text { Tseng y Piller } \\
\text { (2003) }\end{array}$ & $\begin{array}{l}\text { Cuatro niveles en } \\
\text { personalización } \\
\text { en masa }\end{array}$ & Niveles & $\begin{array}{l}\text { 13. Nivel diferenciación. } \\
\text { 14.Nivel coste. } \\
\text { 15.Nivel espacio solución. } \\
\text { 16.Nivel relación. }\end{array}$ & Perspectivas \\
\hline $\begin{array}{l}\text { Blecker et al. } \\
(2005)\end{array}$ & $\begin{array}{l}\text { Sistemas de } \\
\text { empresas } \\
\text { personalizadoras }\end{array}$ & Procesos & $\begin{array}{l}\text { 17.Subproceso desarrollo } \\
\text { 18.Subproceso interacción } \\
\text { 19.Subproceso compra } \\
\text { 20.Subproceso producción } \\
21 . \text { Subproceso logístico } \\
22 \text {.Subproceso información }\end{array}$ & Subsistemas \\
\hline
\end{tabular}

Tabla 2. Resumen de la revisión sobre el proceso de personalización en masa 
El primer paso de la revisión de la literatura que se realiza, será la descripción y análisis de los diferentes modelos en orden cronológico. En la Tabla 2 se resumen las diferentes aproximaciones.

La metodología desde la perspectiva del desarrollo del producto para ofrecer una Arquitectura de Producto (PFA) para la personalización en masa de Jiao y Tseng (1999) ofrece la definición de la plataforma de producto que proporciona la base tecnológica para desarrollar la personalización y potenciar las competencias existentes en la compañía. La importancia del desarrollo de producto para el éxito de cualquier empresa es ampliamente reconocida en la literatura (Henard \& Szymanski, 2001; Christensen, 2013), en el caso de la personalización en masa es incluso más importante y determinante (Lampel \& Mintzberg, 1996; Franke, Keinz \& Schreier, 2008). La arquitectura de producto proporciona la estructura de producto, determinando las diferentes variantes, describiendo las interrelaciones entre ellas y su congruencia con los requerimientos de los clientes y procesos. El estudio realizado por Jiao y Tseng (1999), se centra en la industria electrónica que basa su diseño en productos centrados en componentes $y$, por tanto, posibilita un diseño altamente modular. La investigación se encuentra sesgada por este hecho, por lo que sus conclusiones tienden a ser restrictivas y enfocadas hacia este tipo producción. Sin embargo creemos que los principios de su metodología son extensibles a otros modelos de proceso y con esta visión más amplia describiremos su metodología.

La metodología para el desarrollo de la arquitectura, permite definir y racionalizar la gama de productos para la personalización en masa, usando una planificación sistemática en tres visiones consecutivas. En la Tabla 3 y Figura 1 describimos cada una de ellas.

\begin{tabular}{|l|l|}
\hline Visión & \multicolumn{1}{|c|}{ Descripción } \\
\hline Vista funcional & $\begin{array}{l}\text { Consiste en sus elementos funcionales y sus interrelaciones. En el caso de } \\
\text { personalización en masa, representa la gama global de variantes de productos y la } \\
\text { percepción del cliente del espectro de estas posibles variantes y opciones. En otras } \\
\text { palabras, representa los requerimientos de los clientes capturados por el portofolio } \\
\text { ofertado de variantes de producto. Las características funcionales del producto son las } \\
\text { que dan respuesta a los requerimientos de los clientes. En esta vista se excluyen } \\
\text { explícitamente la ingeniería y los costes. }\end{array}$ \\
\hline Vista técnica & $\begin{array}{l}\text { Representa la solución tecnológica y la aplicación de la tecnología al producto. Por } \\
\text { ejemplo definiría la modularidad de los componentes y las interconexiones entre } \\
\text { módulos. En el modelo técnico se definirían los parámetros de diseño que } \\
\text { corresponderían con los requerimientos funcionales establecidos en la fase anterior. }\end{array}$ \\
\hline Vista física & $\begin{array}{l}\text { Descripción de la realización y construcción del producto. En otras palabras, define } \\
\text { como se va a fabricar el producto, el proceso y la tecnología. }\end{array}$ \\
\hline
\end{tabular}

Tabla 3. Metodología para el desarrollo de la arquitectura del producto (Jiao \& Tseng, 1999) 


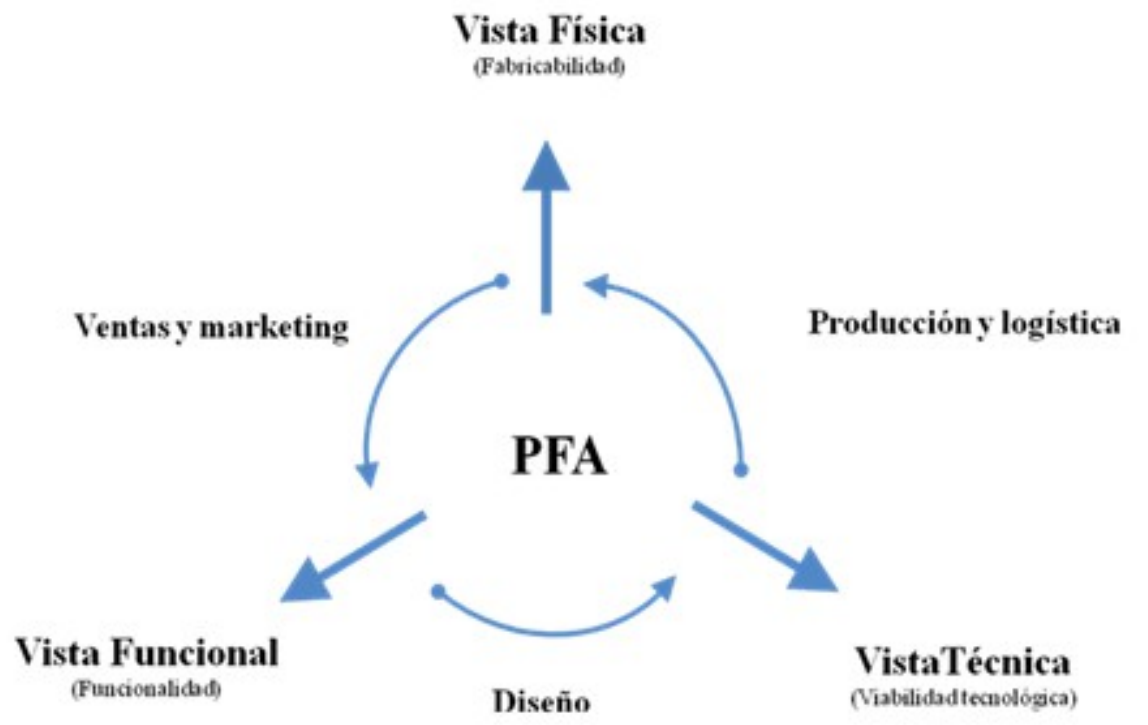

Figura 1. Metodología para el desarrollo de la arquitectura del producto (Jiao \& Tseng, 1999, pp. 6)

En este modelo, el proceso de definición de la gama de productos parte de la vista funcional, delimitando los requerimientos funcionales de los clientes que en principio vamos a satisfacer. La variedad de productos y sus atributos estarán relacionados con una funcionalidad asociada a un requerimiento del cliente. Mediante el proceso de diseño del producto se definen los parámetros o características del producto que satisfacen estos requerimientos y atributos. El segundo paso es buscar la solución tecnológica para el producto. El tercero es definir la solución de producción, proceso, logística y cadena de suministro que satisface las condiciones anteriores, buscando usar las capacidades, competencias de la empresa, economías de escala, reúso de componentes, etc. Por último cerramos el bucle comprobando que la vista física responde a la vista funcional inicial que habíamos definido y en consecuencia al diseño inicial que hemos realizado para nuestro producto, esto se realiza comparando la vista física y la funcional.

El resultado de la aplicación de esta metodología es la definición de la plataforma de producto que proporciona la gama de productos, la base tecnológica y de proceso para desarrollar la personalización. Esto corresponde a la definición del espacio solución, que como hemos señalado anteriormente, es una característica de personalización en masa (Pine 1995). El enfoque de Jiao y Tseng (1999) queda restringido y no establece con claridad los pasos anteriores y posteriores a esta parte del proceso. Por ejemplo, la vista funcional necesariamente tiene que partir de una información conocida sobre el cliente. El proceso de recogida de esta información no se contempla ni se menciona, tampoco se considera la gestión de los pedidos individuales ni su entrega. 
Du, Jiao y Tseng (2006) en investigaciones posteriores sobre la función de utilidad para los clientes, costes y generación de valor en la personalización del producto extienden este modelo e incorporan otros elementos. Sin embargo en ambas investigaciones el modelo de proceso queda incompleto al no formular el proceso de codiseño con el cliente, que hemos identificado como indispensable en la definición de la personalización en masa.

Reichwald et al. (2000) desarrollan desde la perspectiva de la información su modelo. Consta de 5 etapas o pasos del proceso de personalización en masa e identifica los flujos de información entre estos. En la Figura 2 se encuentra la representación del modelo.

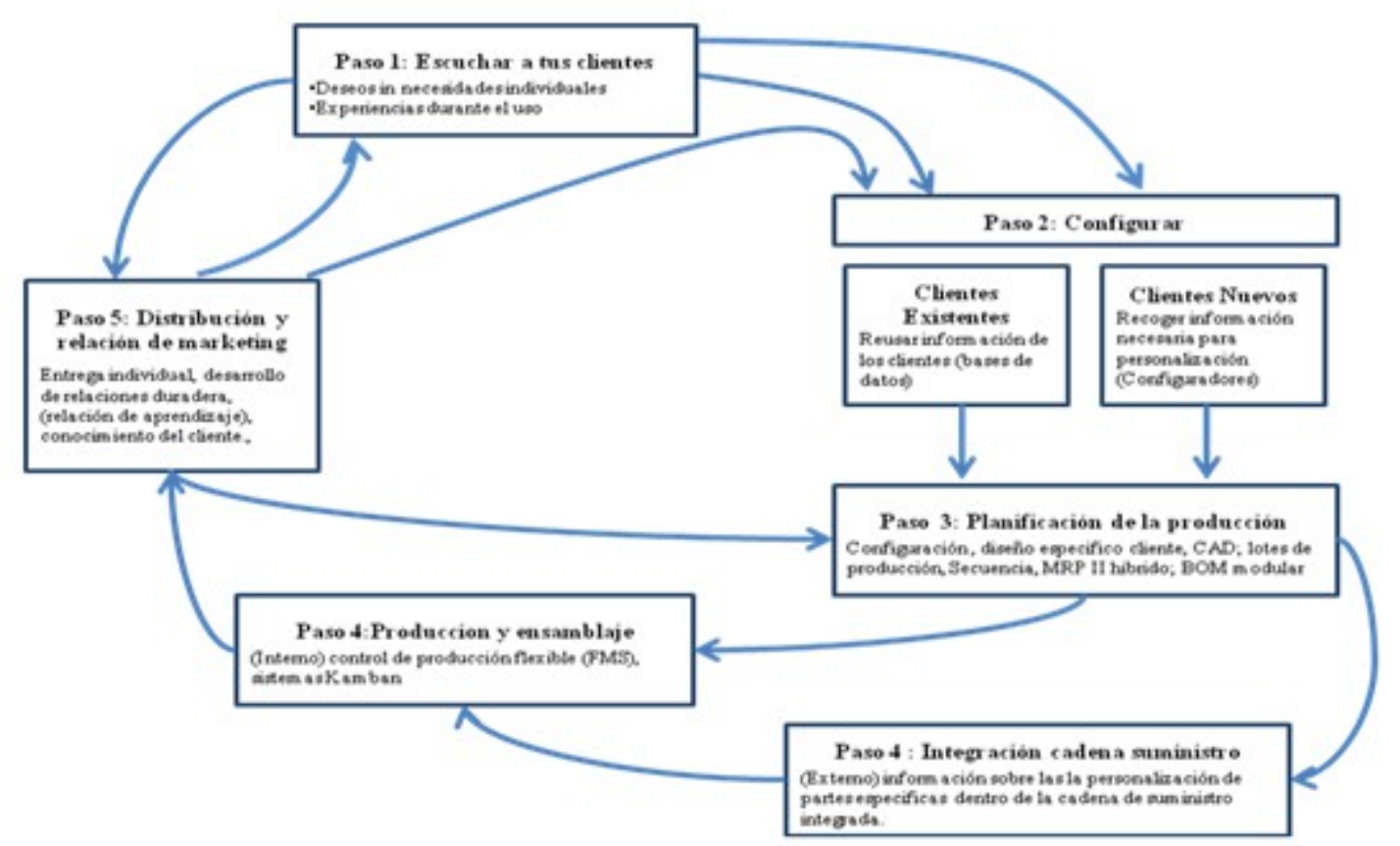

Figura 2. Ciclo de información de la personalización en masa (Reichwald et al., 2000)

El modelo no define con claridad el tipo de relación con el cliente que existe en el paso 1 . No está claramente delimitado si se refiere a una relación individual o a una relación agregada. Éste es uno de los elementos menos claros que en general hemos encontrado en la literatura sobre personalización en masa. Sin embargo la relación individual con el cliente queda claramente identificada en el paso 2 . El diseño del espacio solución no está presente en este modelo, elemento fundamental del proceso y que hemos descrito anteriormente. Teniendo en cuenta que el modelo sólo representa los flujos de información, esta ausencia podría estar justificada. Los pasos tres, cuatro y parte del cinco representan las operaciones de producción y distribución del producto. En el paso 5 se incluye la relación de marketing. El modelo define los flujos de información que parten de este paso hacia el primer, segundo y tercer paso. En 
este paso 5, la relación con el cliente permite recoger información relevante sobre las tendencias del mercado, el valor y la utilidad. Esta información permite refinar, mejorar y evolucionar las premisas iniciales de valor y utilidad, también dará a conocer la idoneidad y potenciales mejoras que se pueden incorporar al espacio solución, lo que facilitará adaptarlo a la demanda real.

Comparando el modelo Reichwald et al. (2000) con el modelo de Jiao y Tseng (1999), se observa que no hay solape en el área analizada por ambos, al estudiar la personalización en masa desde diferentes ángulos, que son complementarios, no excluyentes y coherentes entre sí.

Esta aproximación contiene muchas similitudes con el modelo siguiente, el Ciclo de conocimiento (Tseng \& Piller, 2003), no en vano comparte a uno de los autores. Siguiendo con nuestro análisis, vamos a estudiar ambos modelos en conjunto. Para Tseng y Piller (2003) el objetivo de la personalización en masa es crear una empresa que integre al cliente, por lo que el alcance del sistema o proceso empleado debe ir más allá de lo que una empresa de fabricación tradicional hace. Para que la empresa pueda ser proactiva, debe incluir la capacidad de detectar las necesidades de los clientes, para posteriormente trabajar sobre estas necesidades, alineando y posicionando la empresa, sus recursos y capacidades hacia los futuros requerimientos y tendencias. Tseng y Piller (2003) definen el ciclo de conocimiento de la empresa, que representa la importancia de ese flujo interconectado e integrado de comunicación y conocimiento, como la columna vertebral de cualquier empresa que busque centrarse en el cliente. En la Figura 3 encontramos la representación gráfica del Ciclo de conocimiento.

Aunque el diseño del espacio solución se encuentra en el inicio del gráfico, los autores señalan la interacción con el cliente como el inicio del proceso. La interacción con el cliente es donde se recoge y analiza la información necesaria para la personalización, etapa que ellos denominan configuración. La etapa de ejecución junto con la de gestión del cliente serán las que garantizarán su fidelidad. El conocimiento generado al proporcionar servicio a un cliente se puede utilizar para servirle mejor y más rápido cuando se produzcan órdenes repetitivas. De la misma forma, durante la ejecución se genera conocimiento que alimentará al diseño del espacio solución, mejorando la eficiencia y calidad. Este conocimiento también puede alimentar a la cadena de suministro para mejorar su eficiencia y eficacia. En este modelo el espacio solución se reconoce claramente como un elemento de la personalización en masa, situándolo al inicio de la representación gráfica pero no al principio del proceso. 


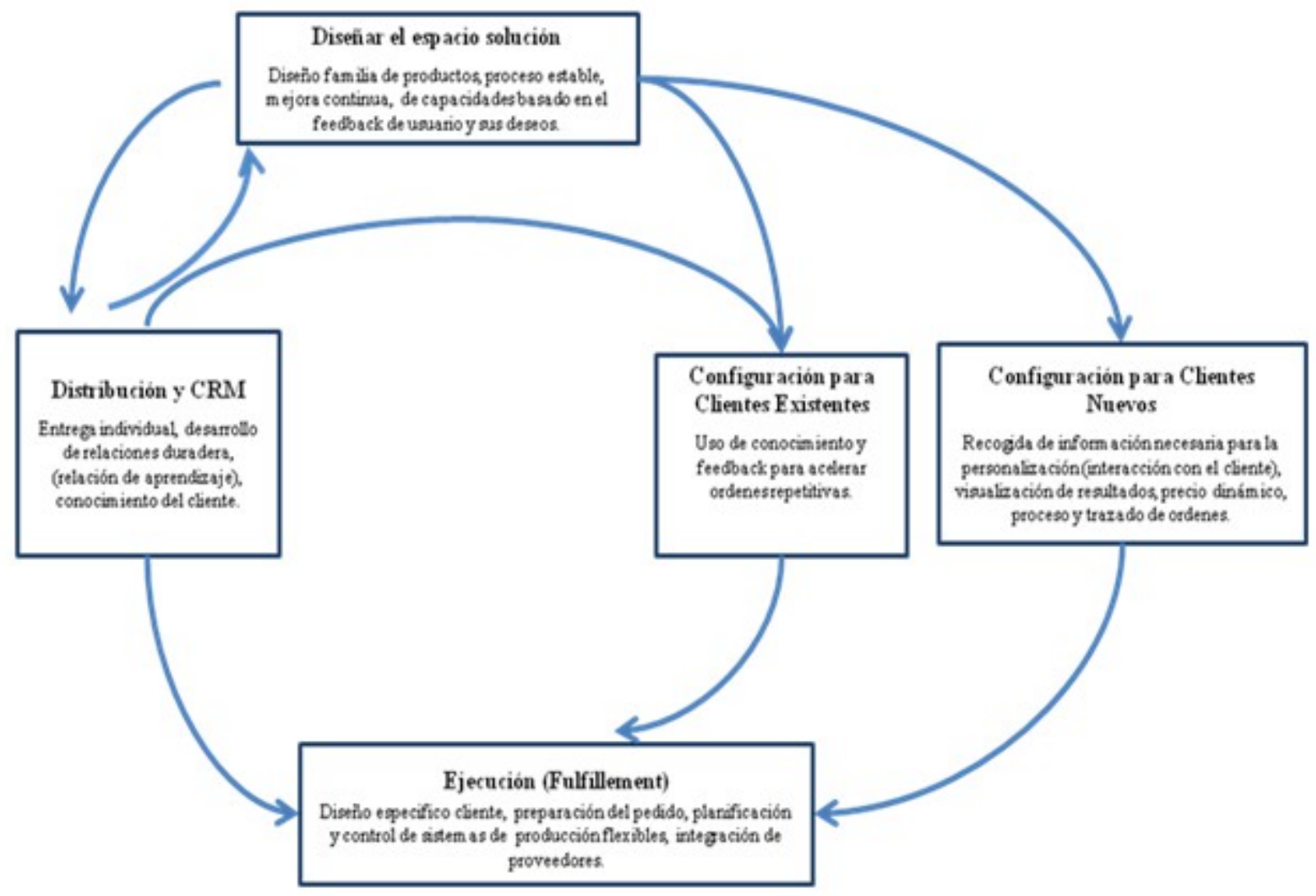

Figura 3. Ciclo de conocimiento de la empresa (Tseng \& Piller, 2003, pp. 11)

Comparando el modelo de Ciclo de información (Reichwald et al., 2000) y Ciclo de conocimiento (Tseng \& Piller, 2003) encontramos tres diferencias. La primera diferencia se localiza en la parte superior del gráfico, en el modelo de ciclo de información aparece como primer paso "escuchar a los clientes", mientras que en el modelo de ciclo de conocimiento en esa posición se encuentra "diseñar el espacio solución". Sin embargo, para ambos modelos los autores afirman que el inicio del proceso es la "interacción con el cliente". La segunda diferencia se encuentra en que en el modelo de Tseng y Piller (2003), el elemento de ejecución (fullfilment) resume los pasos 3 y 4 del otro modelo: planificación de la producción, integración de la cadena de suministro, producción y embalaje. La tercera es que el primer modelo representa los flujos de información y el segundo de conocimiento.

En este punto debemos distinguir entre conocimiento e información. Reichwald et al. (2000) describen flujos de información y Tseng y Piller (2003) definen los mismos flujos entre las mismas etapas, pero los consideran conocimiento. Las mínimas diferencias que se pueden observar en los flujos de ambos modelos son debidas a que Tseng y Piller (2003) han simplificado agrupando las etapas y en consecuencia también han eliminado parte de los flujos, en concreto la retroalimentación a la etapa de ejecución desde la etapa de CRM. Los 
flujos de información se generan por y como necesidad de las operaciones, la información del proceso se recogerá del cliente, preferentemente apoyada por las tecnologías de la información y se transmitirá de esta misma forma a los diferentes pasos. Lo que Tseng y Piller (2003) no describen es como esos flujos de información se convierten en conocimiento para la empresa. Información no es lo mismo que conocimiento (Davenport \& Prusak, 1997) y para que exista generación de conocimiento es necesario contar con los instrumentos y procesos que transformen la información en conocimiento.

Ambos modelos no incluyen el flujo de materiales y su proceso no es completo, pero si incluyen elementos claves. Con una perspectiva más amplia, el modelo de Tseng y Piller (2003) incluye el diseño del espacio solución mientras que el modelo Reichwald et al. (2000) se centra en la parte final del modelo, el co-diseño con el cliente y la entrega del producto. Consideramos que ambos modelos son coherentes y complementarios.

\begin{tabular}{|l|l|}
\hline \multicolumn{1}{|c|}{ Nivel } & \multicolumn{1}{c|}{ Descripción } \\
\hline Diferenciación & $\begin{array}{l}\text { Basada en la utilidad adicional que obtiene los clientes adquiriendo servicios que } \\
\text { se adaptan mejor a sus necesidades. }\end{array}$ \\
\hline Coste & $\begin{array}{l}\text { Utilizando las técnicas que hacen que los costes se mantengan a nivel próximo a } \\
\text { producción en masa. }\end{array}$ \\
\hline Espacio de solución & $\begin{array}{l}\text { Definición de la solución operativa y organizativa que proporciona la flexibilidad } \\
\text { manteniendo la respuesta. }\end{array}$ \\
\hline Relación & Estableciendo la relación y co-diseño con el cliente. \\
\hline
\end{tabular}

Tabla 4. Niveles de personalización en masa (Tseng \& Piller, 2003)

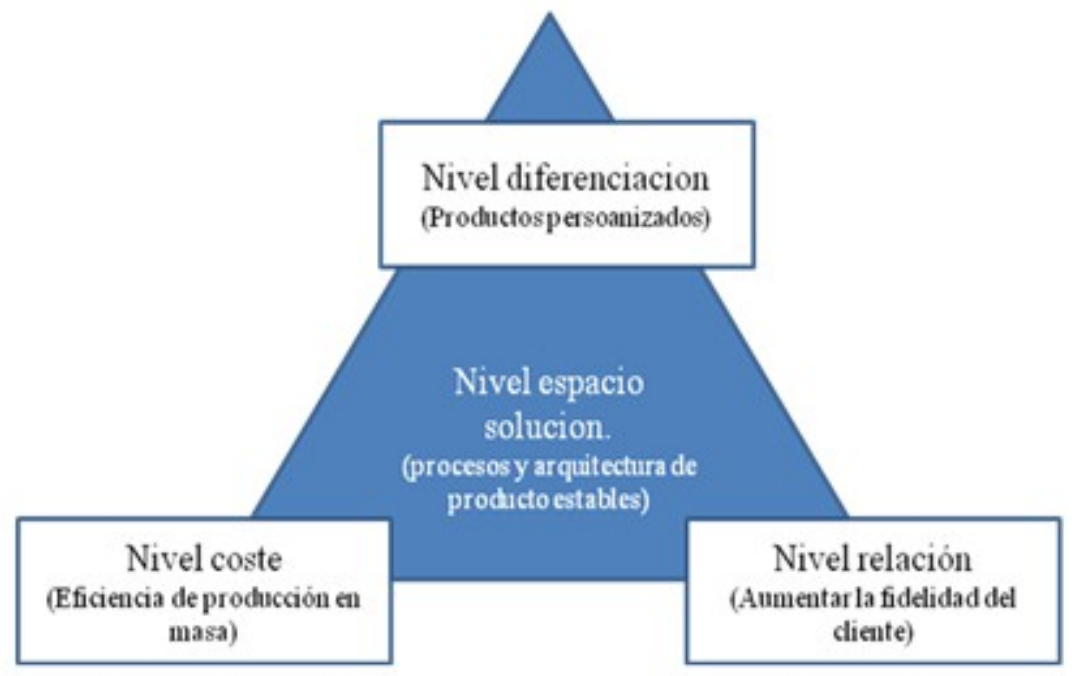

Figura 4. Cuatro niveles de personalización en masa (Tseng \& Piller, 2003, pp. 6) 
Tseng y Piller (2003) en el mismo artículo que contiene su Ciclo de conocimiento afirman que la personalización en masa se establece en cuatro niveles. La Tabla 4 describe estos niveles. La palabra nivel corresponde a la traducción directa, sin embargo perspectiva o ángulo podrían ser traducciones más adecuadas.

En estos niveles encontramos los objetivos que se persiguen. En la Figura 4 se reflejan los elementos con los que se esperan alcanzar los objetivos.

En esta aproximación los autores no separan la relación y los flujos de información que se generan en la interacción con el cliente. Con esta información podemos asociar cada uno de estos niveles a los elementos del modelo que se va a proponer en este documento (etapas y bucles). El nivel diferenciación corresponde a la identificación del valor y utilidad. El nivel espacio solución correspondería al diseño del espacio solución, el nivel coste corresponde al bucle de validación que contempla la generación de valor tanto para el cliente como para la empresa. Por último, el nivel de relación se refiere al co-diseño con el cliente y la entrega.

Blecker et al. (2005) analizan el proceso de personalización en masa desde la perspectiva de sistemas y subsistemas. Estos subsistemas realizan cada uno de los subprocesos del proceso general de personalización en masa. Los autores definen el conjunto de actividades interrelacionadas necesarias para capturar los requerimientos individuales y transformarlos en el producto que se entrega al cliente, dividiendo el proceso en seis subsistemas. En la Tabla 5 se describen brevemente cada uno de estos subprocesos.

Por último, Blecker et al. (2005) representan el proceso de forma lineal comenzando en el subproceso de desarrollo y continuando secuencialmente hasta el subproceso logístico. El subproceso de información apoya cada una de estos subprocesos. El primer subproceso, el de desarrollo, incluye la definición del espacio solución aunque no incluye el diseño de la cadena de suministro o de los medios productivos, es decir se limita a la definición del producto. El modelo de Jiao y Tseng (1999) es más amplio y si contempla el diseño de los medios para poder suministrar el producto. Para Blecker et al. (2005), el espacio solución se limita por tanto, al desarrollo de producto una vez definidos todos los sistemas de producción, logística y compra. El subproceso de interacción corresponde al codiseño. Los subprocesos de compra, producción y logística corresponden a la ejecución de la orden, apoyan la fase de entrega al cliente y en su definición no difieren de lo descrito en los modelos anteriores. El proceso de información facilita las operaciones pero no describe la adquisición de ningún conocimiento o recogida de información sobre el cliente, sólo es el soporte para las operaciones. El subproceso de información no incluye explícitamente las interacciones y bucles de retroalimentación. Comparando este modelo con los analizados anteriormente no observamos ninguna contradicción. La representación gráfica del modelo se encuentra en la Figura 5. 


\begin{tabular}{|c|l|}
\hline SUBPROCESO & \multicolumn{1}{|c|}{ DESCRIPCIÓN } \\
\hline Desarrollo & $\begin{array}{l}\text { Su función es trasladar las necesidades generales del cliente objetivo a una } \\
\text { arquitectura de producto de la que se pueden obtener un amplio número de } \\
\text { variantes de producto. En este proceso, los ingenieros de diseño definen los grados } \\
\text { de libertad que los clientes pueden utilizar para crear su variante individualizada. }\end{array}$ \\
\hline Interacción & $\begin{array}{l}\text { Consiste en la recogida de la información y necesidades del cliente, asociándolas al } \\
\text { producto más apropiado. Este proceso se puede apoyar en Internet o a través de } \\
\text { minoristas. }\end{array}$ \\
\hline Compra & $\begin{array}{l}\text { Debido a la flexibilidad necesaria para la personalización en masa, el outsourcing es } \\
\text { un elemento muy utilizado. Este subproceso se encarga de gestionar las relaciones } \\
\text { con los proveedores y su selección. }\end{array}$ \\
\hline Producción & $\begin{array}{l}\text { Encargado de la producción y fabricación del producto. Son los medios productivos } \\
\text { que deberán ser flexibles para dar respuesta a demandas de la personalización en } \\
\text { masa. La modularidad se describe como un elemento clave de este subsistema, pero } \\
\text { no imprescindible. }\end{array}$ \\
\hline Logístico & $\begin{array}{l}\text { Incluye el flujo logístico tanto aguas arriba como aguas abajo. Los autores destacan } \\
\text { el diferencial de coste que la logística de entrega al cliente presupone, debido a que } \\
\text { al ser personalización en masa, las entregas son individualizadas y por lo tanto } \\
\text { menos eficientes que los sistemas tradicionales de distribución. }\end{array}$ \\
\hline Información & $\begin{array}{l}\text { Interactúa con todos los procesos anteriores, facilitando la información necesaria. } \\
\text { Desde capturar las necesidades del cliente, definir la lista de requerimientos del } \\
\text { producto, la ruta de producción, hasta preparar el envío e incluso el seguimiento de } \\
\text { la orden. }\end{array}$ \\
\hline
\end{tabular}

Tabla 5. Subprocesos relevantes para la personalización en masa (Blecker et al., 2005)

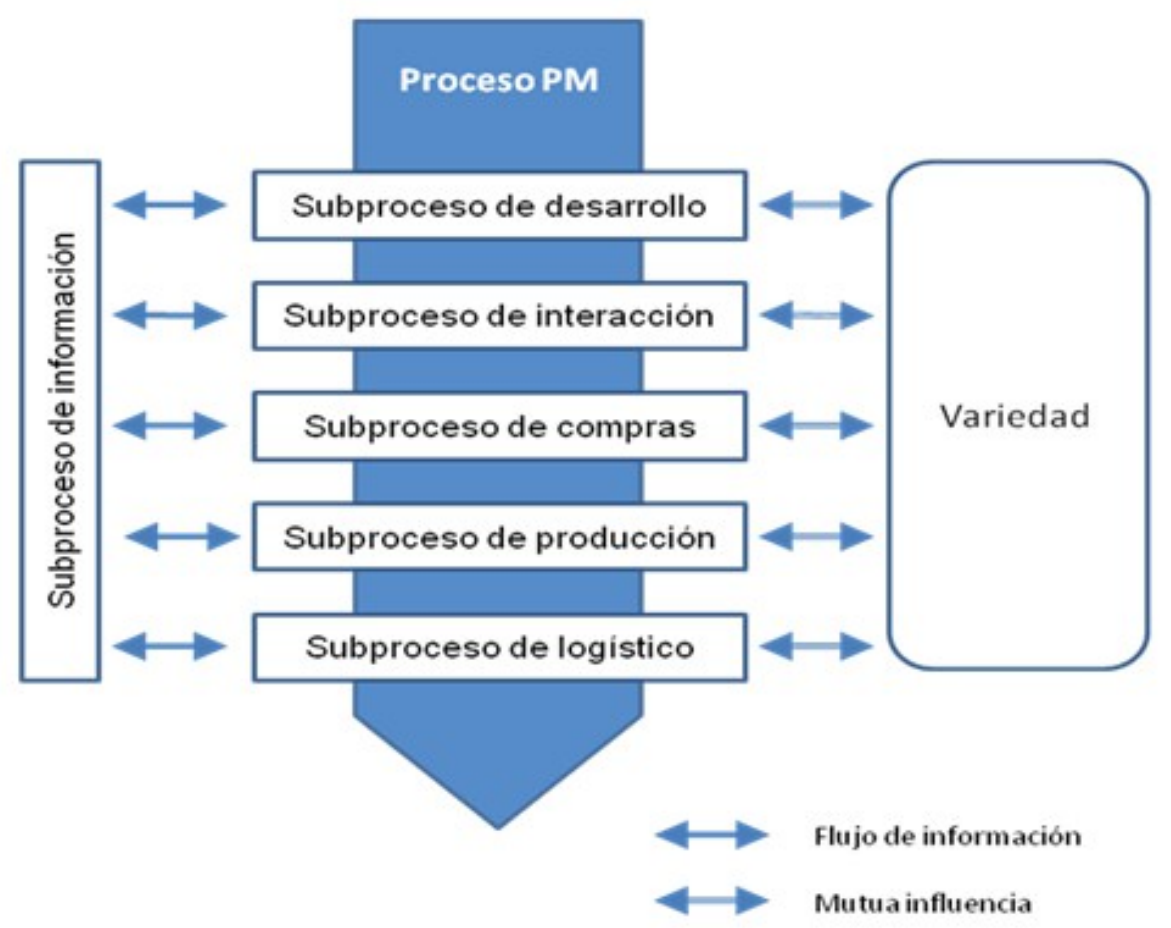

Figura 5. Subprocesos relevantes para la personalización en masa (Blecker et al., 2005, pp. 189) 
Del análisis realizado en este apartado concluimos que las investigaciones previas que desarrollan aproximaciones al modelo de la personalización en masa, lo hacen desde diferentes perspectivas: diseño de producto, información, conocimiento, niveles, sistemas y subsistemas. Ninguno de los autores ofrece un modelo de personalización en masa desde la perspectiva global del negocio. Identificado este hueco en la literatura y partiendo del análisis aquí realizado, en el siguiente apartado complementaremos estas aproximaciones y propondremos un modelo general de negocio para la personalización en masa.

\section{Modelo de negocio para la personalización en masa}

Para implementar esta estrategia parece necesario orientarse en un modelo de negocio; ya que, los modelos de negocio representan la forma en que las empresas realizan sus negocios, como se organizan, los flujos de materiales e información, los recursos que utilizan y las propuesta de valor a los clientes (Amit \& Zott, 2001). También permiten tanto a directivos como académicos, describir y distinguir los diferentes tipos de actuación de las compañías, así como entender mejor los elementos y las claves.

En la Figura 6 se encuentra el modelo de negocio que se propone para la personalización en masa.

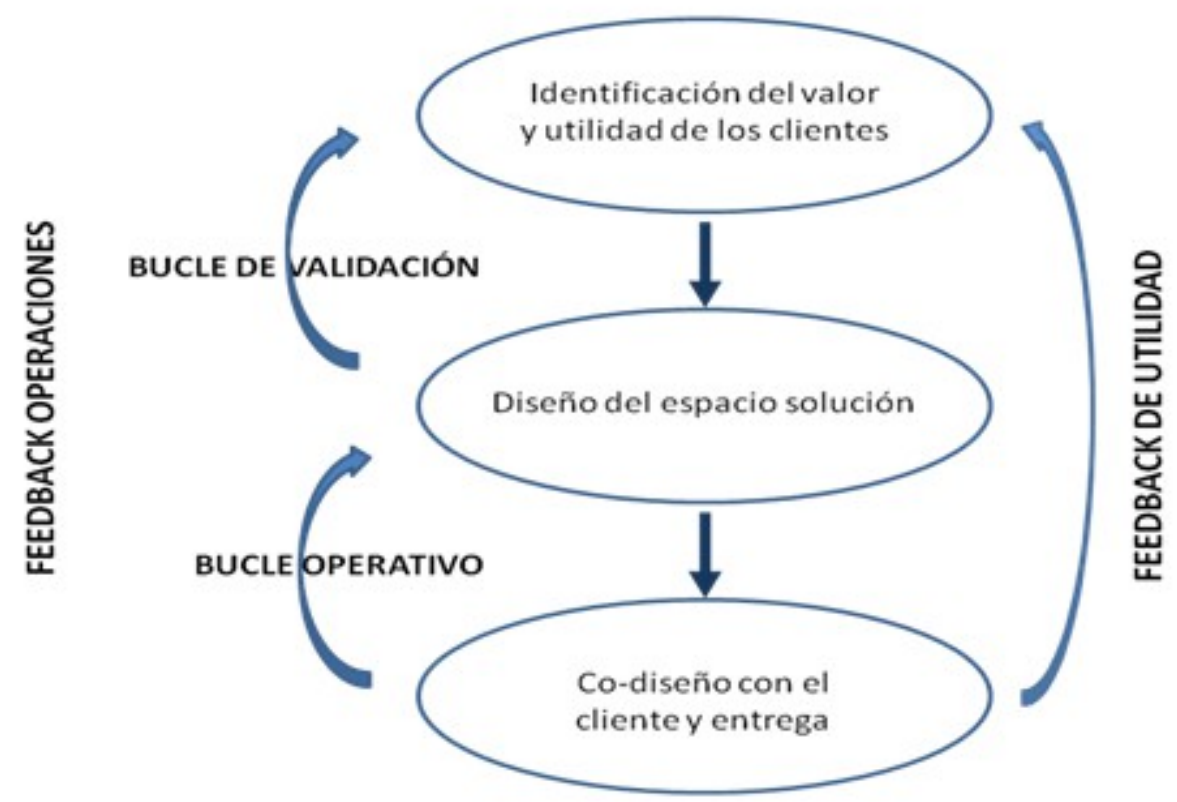

Figura 6. Modelo de negocio de la personalización en masa 
El modelo propuesto se compone de tres etapas: identificación del valor y utilidad para los clientes, diseño y definición del espacio solución y co-diseño con el cliente y entrega y de tres bucles de información: bucle económico de validación, retroalimentación de utilidad y retroalimentación operativa. A continuación, se comentan brevemente cada una de las etapas identificadas.

La primera etapa del modelo de negocio es determinar las necesidades y preferencias del cliente, es decir identificar su valor y utilidad. Para ello, hay que identificar la oportunidad de demanda insatisfecha en el mercado que se quiere cubrir mediante la personalización. Este principio no es diferente a otros negocios, la particularidad de la personalización en masa en este punto es que la demanda insatisfecha se produce como consecuencia de las necesidades individuales no cubiertas por las características generales de los productos estándar. En este primer paso se analizan y clasifican las necesidades individuales a nivel agregado, definiendo los generadores y parámetros de personalización y como éstos producen utilidad para los clientes. Esta fase será la que se desarrollaron más detalle en este artículo.

La propuesta de valor al cliente tiene que materializarse, este es el siguiente paso del diseño y definición del espacio solución El espacio solución representa el marco en que la empresa va a trabajar: la oferta de producto (diseño, características personalizables, familia de productos, variantes), el proceso (modularidad, tecnología, flexibilidad, sistemas de información....) y las operaciones (cadena de suministro, planificación y organización), para alcanzar el doble objetivo de coste y personalización. El espacio solución es una característica de personalización en masa y es un proceso estable pero a la vez flexible y capaz de responder y suministrar un flujo dinámico de productos (Pine, 1995). Pero al mismo tiempo, el espacio solución limita la oferta de producto, variantes y personalización, que será finita, en la misma forma que los recursos, capacidades y tecnologías disponibles lo son para la empresa. Se profundizará más en esta etapa en el apartado 4.1.

La empresa que implementa la personalización en masa espera obtener un sobreprecio superior al coste que supone la personalización (Spring \& Dalrymple, 2000) ya que los clientes generalmente estarán dispuestos a pagar un sobreprecio por productos que satisfagan mejor sus necesidades individuales (Roberts \& Meyer, 1999). Cualquier empresa que quiera aplicar diferenciación o personalización a un producto deberá asegurarse que los beneficios adicionales de aplicar la personalización al producto para el cliente, sean superiores al sobreprecio que demanda.

En la fase de diseño de cualquier producto, se definen el proceso, la calidad y se delimitan prácticamente todos los costes de generar el producto. En la personalización en masa esto ocurre cuando definimos el espacio solución. En consecuencia, el coste de producción y las 
características del producto vienen ambos determinados por el espacio solución. En el diseño del espacio solución es fundamental considerar y analizar las economías de escala, alcance y aprendizaje que deberán preservarse y potenciarse para alcanzar los objetivos de costes. Una vez concretado el espacio solución, se obtendrá la propuesta de valor y los costes asociados, que deberán cumplir los objetivos de coste para poder ofertar precios competitivos.

El siguiente paso debería ser validar el espacio solución, validez en términos de rentabilidad y generación de valor. Hay que comprobar si la oferta de personalización seleccionada genera valor en los siguientes términos:

- Valor para el cliente, de tal forma que la utilidad marginal del consumidor sea superior al sobreprecio que tiene que pagar por el producto personalizado.

- Valor para la empresa, que el precio sea superior al coste de generación del producto.

Es decir, en términos relativos, el sobrecoste de producir el producto personalizado deberá ser inferior al sobreprecio que permite el mercado sobre el estándar. Este análisis es el que hemos denominado bucle económico de validación. El bucle de validación debería incluir varias iteraciones, en las que se exploren diferentes familias y configuraciones de productos, diseños, procesos, etc. En definitiva diferentes espacios solución y sus costes asociados, comparándolos con los diferentes valores que estos regeneran para el cliente y los precios que estarían dispuestos a pagar, para así obtener el espacio solución que, en principio, maximice los beneficios.

Una vez definido e implantado el espacio solución definitivo, se estará en condiciones de comercializar el producto y ejecutar la tercera etapa del proceso, el codiseño con el cliente y la entrega. El proceso de personalización requiere que el cliente individual transfiera sus requerimientos y necesidades a una especificación concreta de producto (Piller, 2005). Estos requerimientos y necesidades se establecerán dentro del espacio solución por el que quedarán limitados. Es un proceso de interacción y cooperación entre la empresa y el cliente individual que caracteriza y diferencia a la personalización en masa de otros modelos como pueden ser la producción ligera y ágil (Piller,2005).

El tool kit es el instrumento que facilita al cliente el diseño del producto (Franke, Von Hippel \& Schreier, 2006), en un proceso de prueba y error, que obtiene un producto altamente personalizado e innovador. Este configurador se apoya en el uso de las tecnologías de la información y debe estar integrado en los sistemas de la compañía para obtener las economías necesarias. 
A su vez el configurador será útil para recoger información de los clientes y crear una estrecha relación con ellos. Esta recogida de información tiene un triple componente, uno interno y dos externos a esta etapa:

- El componente interno tiene un enfoque CRM, la información recogida permitirá conocer mejor las necesidades, preferencias, costumbres y hábitos de consumo de cada cliente individual, haciendo posible el diseño e implementación de marketing individualizado o uno-a-uno, al mismo tiempo que mejorará el servicio y perspectivas de venta.

- En los componentes externos, la información alimenta a las otras dos fases o etapas del modelo. Por una parte la recogida de información permite conocer la idoneidad y potenciales mejoras que se pueden incorporar al espacio solución, que permitirá refinarlo mejorarlo y adaptarlo a la demanda real. Este bucle de información se denomina retroalimentación operativa. Por otra parte, el proceso de codiseño nos permite recoger información relevante sobre las tendencias del mercado y clientes, el valor y la utilidad. Incluso en toolkits mas avanzados podemos tener la posibilidad de que los clientes innoven los diseños genéricos de espacio solución. Esta información va a permitir refinar, mejorar y evolucionar las premisas iniciales de valor y utilidad para el cliente. A este bucle de información se denomina retroalimentación de utilidad.

Cada una de las distintas etapas se relacionan con el entorno y más concretamente, con los clientes y la cadena y sistema de valor de la empresa. En la fase de identificación de valor y utilidad de los clientes, la relación con los clientes es una necesidad, el sistema se relaciona con los clientes a nivel agregado para definir el valor para estos. En el diseño del espacio solución, la empresa se relaciona e interactúa con su cadena y sistema de valor para definir la propuesta de valor de la empresa. Y en la última etapa la empresa se relaciona e interactúa con ambos agentes, con los clientes individuales para recoger sus necesidades individuales y con el sistema de valor para materializar el producto.

\subsection{Diseño del espacio de soluciones para personalizar en masa}

\subsubsection{Propuesta de valor}

Uno de los retos más importantes de la primera etapa de la personalización en masa es la de definir los atributos del producto que generen valor para el cliente. Los atributos de un producto son sus características personalizables a ofertar. Estos atributos formarán el espacio solución, y serán los generadores de valor de la personalización en masa. 
En la literatura se han encontrado cuatro tipos de generadores definidos por diferentes autores (Tabla 6).

\begin{tabular}{|c|c|c|c|}
\hline GENERADOR & Piller (2005) & $\begin{array}{c}\text { McCahrty } \\
\text { et al (2006) }\end{array}$ & $\begin{array}{c}\text { Franke y Schreier } \\
\text { (2008) }\end{array}$ \\
\hline AJUSTE FÍsICO & $\begin{array}{c}\text { Medida } \\
\text { (ajuste y confort) }\end{array}$ & $\begin{array}{c}\text { Dimensión física o } \\
\text { ajuste }\end{array}$ & \\
\hline FUNCIONALIDAD & Funcionalidad & $\begin{array}{c}\text { Hardware } \\
\text { Software } \\
\text { Propiedades } \\
\text { Grado } \\
\text { Nivel de calidad } \\
\text { Literatura } \\
\text { Embalaje }\end{array}$ & Funcional \\
\hline AJUSTE ESTÉTICO & $\begin{array}{c}\text { Estilo } \\
\text { (diseño estético) }\end{array}$ & Estética o estilo & Estético \\
\hline EXCLUSIVIDAD & & Personalización & Exclusividad \\
\hline
\end{tabular}

Tabla 6. Clasificación de las características del producto

Piller (2005) define tres dimensiones del producto en las que se basa la personalización en masa: medida (ajuste físico), funcionalidad y estilo (ajuste estético). La dimensión medida, ajuste y confort, establecen como personalizar el producto adaptándolo a medidas físicas, como puede hacerse con un traje o un mueble. Es la dimensión más difícil para implementar y requiere de alta interacción con el cliente. La dimensión de funcionalidad es la personalización de los atributos técnicos o funcionales del producto. Aunque es más fácil de implementar, es la que menos se utiliza en el proceso de personalización en masa. Siguiendo a este autor, el estilo se definiría como las modificaciones del producto que se dirigen a aspectos sensoriales $u$ ópticos. Considera dentro de esta dimensión que una parte del valor generado corresponde al factor de exclusividad, es decir a la búsqueda de individualidad que persiguen un pequeño número de clientes.

Aunque Piller (2005) afirma que el ajuste estético y la exclusividad no producen utilidad para un porcentaje suficiente de la población para generar valor, Franke y Schreier (2008) afirman que la exclusividad percibida si genera valor para toda la población. Ambos afirman que la exclusividad genera valor para el cliente, en lo que discrepan es en la cuantía de valor generado.

Por su parte, MacCarthy, Brabazon y Bramham (2006) en un estudio de cinco productos que aplican la personalización en masa: bicicletas, ordenadores, componentes de comunicación, teléfonos móviles y vehículos comerciales, concluye que existen 10 atributos. Estos atributos proporcionan un mayor nivel de detalle y puedan ser reclasificados dentro los grupos definidos por los autores anteriores. 
Observamos en la Tabla 6, que se pueden agrupar los atributos de las tres investigaciones en cuatro dimensiones genéricas, que definiremos como cuatro generadores de diseño (primarios) de la personalización en masa: ajuste estético, ajuste físico, funcionalidad y exclusividad siendo estos los que guardan relación directa con las cualidades del producto y por tanto están orientados a su diseño y son los que realmente determinan el espacio de soluciones. A continuación los definiremos con mayor precisión.

El ajuste físico consiste en adaptar el producto a las dimensiones físicas que requiere el cliente. Estas dimensiones físicas pueden ser dimensiones humanas (altura, cintura...) o materiales y de espacio.

La funcionalidad es ajustarse a los parámetros funcionales requeridos por el cliente como: potencia, velocidad, peso, anchura de corte, diámetro de taladro, etc... Esta dimensión es muy utilizada en la personalización de productos industriales, en que los clientes necesitan unas especificaciones definidas para cubrir sus necesidades concretas. Es una dimensión sobre la que el cliente industrial tiene un mejor conocimiento y definición de sus necesidades, por ello las empresas suministradoras pueden caracterizar los clientes y agruparlos en función de estas necesidades. Esta dimensión también existe en los productos de consumo. Pero si bien el comprador de productos industriales suele conocer las funcionalidades que necesita y sabe traducirlas a componentes u opciones del producto, en el caso del comprador de bienes de consumo esto no es totalmente cierto, los consumidores finales establecen las características del producto en términos de necesidades y no de componentes.

El ajuste estético es la satisfacción de las preferencias de color, estilo, sabor, olor... en general, requerimientos estéticos y sensoriales. Es un factor completamente intangible y dependerá fundamentalmente de los gustos del cliente.

La exclusividad cubre la necesidad del cliente de diferenciarse utilizando productos exclusivos.

Además de estos generadores, la observación del mercado apunta hacia otros generadores, que denominaremos los generadores de mercado (secundarios), que influyen en la decisión final del cliente sobre la opción del producto a escoger y por tanto, se orientan al mercado y resultan de la percepción del cliente sobre la combinación de los anteriores. Los generadores secundarios identificados son dos: el ajuste económico y el ajuste por la novedad del producto. El ajuste económico se centra en cómo el cliente percibe el valor del producto, mientras que el ajuste por la novedad del producto se centra en la percepción del cliente en la introducción de nuevos productos. En la Figura 6 aparecen representados todos los generadores. 


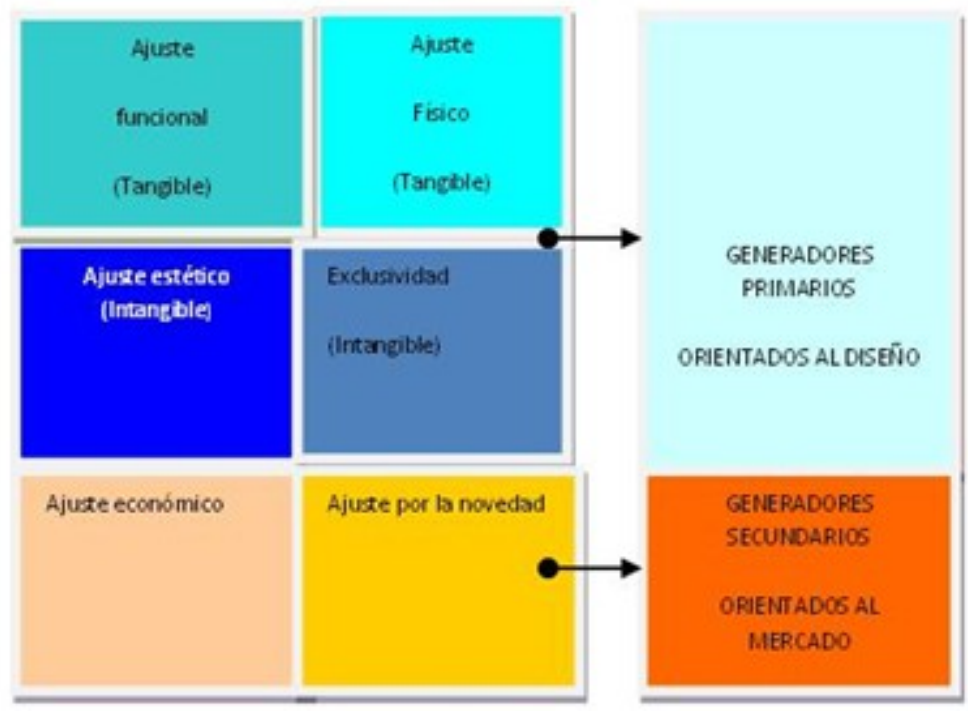

Figura 6. Los generadores de la personalización en masa

El ajuste económico es posible cuando el proceso de co-diseño establezca los precios del producto en función de las opciones escogidas e incorporadas. Ante esta posibilidad, el cliente maximizará su utilidad, escogiendo únicamente aquellas opciones que le generen un excedente de valor en relación al precio. Si el configurador establece un precio fijo con independencia de las opciones escogidas, el ajuste económico no será posible para el cliente, de esta forma la empresa tiene control sobre este generador.

Encontramos ejemplos de ajuste económico en el sector servicios, en concreto en los seguros. AXA ha lanzado en 2010 su seguro AutoFlexible, (http://www.axa.es) un seguro para coches en que se pueden seleccionar un conjunto de coberturas adicionales y personalizadas, en que el cliente sólo cubrirá y pagara por las coberturas que necesite. En este tipo de producto el espacio fijo solución permite una correlación casi de uno entre el coste variable directo y el precio. La competencia en este generador puede ser viable cuando existe una estructura de costes donde predominan los fijos sobre los variables directos, como en el caso de productos digitales y ciertos servicios.

El ajuste económico se puede aplazar en el tiempo. En el caso de la joyería Pandora (www.pandora.net) se produce este tipo de ajuste. El cliente puede fijar el nivel de gasto que va a realizar en el tiempo, partiendo de un gasto inicial que puede ser tan pequeño como el engarce, y puede ir comprando más tarde los diferentes abalorios hasta completar la pulsera o collar. Incluso puede hacer participar a otras personas en el gasto, pidiendo que le regalen componentes por Navidad, cumpleaños u otros eventos. Desde la perspectiva de la empresa esto le genera valor, ya que incrementa el dinero disponible para la compra del producto. 
El segundo generador secundario es el ajuste por la novedad del producto, que se centra en la percepción del cliente cuando se introduce un nuevo producto. Cuando se lanza un nuevo producto, éste genera interés en los clientes atrayéndolos. Si finalmente se acepta por la mayoría, se convertirá en un producto estable, aunque otra posibilidad es que sólo atraiga a un número pequeño de clientes que buscan diferenciarse y dure muy poco tiempo. Esto ocurre cuando el producto no cubre una necesidad real o no lo hace de forma adecuada (Kotler \& Armstrong, 1991). Tenemos numerosos ejemplos de esta situación en la personalización en masa, como pueden ser las tazas personalizadas con la foto, chocolates con mensaje impreso, libros con personajes con tu nombre, etc. Todos ellos solo crean una primera impresión y estos productos tenderán a no tener compras repetitivas.

Por último, destacar que aunque en el análisis realizado anteriormente se ha considerado que la personalización generaba valor para el cliente sin ningún tipo de restricción, hay que tener en cuenta que el cliente no busca personalización per se, no quiere variedad, quiere satisfacer necesidades particulares. En otras palabras, el objetivo de la empresa debe ser encontrar y proporcionar las características personalizables del producto relevantes para el cliente y en la cantidad precisa.

\subsection{Propuesta productiva}

El espacio solución determina la oferta de producto (diseño, características personalizables, familia de productos, variantes), el proceso (equipos, tecnología, flexibilidad, sistemas de información, gestión de calidad, etc.) y las operaciones (cadena de suministro, planificación, organización). Para alcanzar el doble objetivo simultáneo de coste y personalización, se incorporan al espacio solución los posibilitadores (enablers), o técnicas que con mayor frecuencia se encuentran en la literatura. Son las siguientes: diseño de producto (QFD) y diseño de la arquitectura de producto, modularidad, técnicas de fabricación flexibles, integración de los sistemas de información y planificación, respuesta (time based).

Ninguna de estas técnicas es de uso exclusivo de la personalización en masa y se pueden encontrar en la producción ágil, en la producción flexible e incluso en la ajustada. Resaltamos dos de las características diferenciadoras del espacio solución frente a estas técnicas. La primera es el resultado que se persigue: proveer de producto personalizado, para ello se trabaja sobre la arquitectura de producto y se retrasa la asignación del producto al pedido del cliente (posponer). La arquitectura de producto permite la plataforma de producto que proporciona la base tecnológica para desarrollar la personalización y potenciar las competencias de la compañía. Posponer permite acercarse hacia una producción bajo pedido 
sin los inconvenientes que esto representa. La segunda característica se deriva de que el espacio solución es finito y como consecuencia se enmarca en el diseño colaborativo con el cliente, siendo el producto resultado del diseño individual, una de las posibles variantes que ya se habrían previsto en la definición original del espacio solución. Por consiguiente, la gestión de variantes tiene un papel preponderante.

\section{Conclusiones}

Ante la ausencia de un modelo de negocio generalmente aceptado para la personalización en masa, en este trabajo se ha definido y desarrollado el proceso general de implantación de la personalización en masa en tres etapas, detallando brevemente cada una de ellas, dedicando un epígrafe para describir los parámetros que generan el espacio solución. También, en este trabajo se ha hecho énfasis en las iteraciones que existen entre las etapas, que convierten este proceso no en algo puramente secuencial, sino retroalimentado.

Del proceso anterior se pude concluir que no es posible considerar la personalización en masa, limitada a una estrategia productiva y un conjunto de técnicas, debido a las interconexiones que mantiene con la propuesta y generación de valor. Es precisamente en este aspecto, la generación de valor, donde el modelo resulta especialmente útil. Cuando se identifica el valor, la particularidad de la personalización en masa frente a otras estrategias, es que la demanda insatisfecha que se busca emerge de las necesidades individuales no cubiertas por las características generales de los productos estándar. Estas necesidades se satisfacen por los atributos personalizables que denominamos los generadores. Se distinguen dos grupos de generadores: los generadores de diseño (primarios) y los generadores de mercado (secundarios). Los generadores de diseño, que guardan relación directa con las cualidades del producto y por tanto están orientados a su diseño, son los que realmente determinan el espacio de solución. Los generadores de mercado (secundarios) influyen en la decisión final del cliente.

El modelo de negocio propuesto considera que la oferta de personalización es finita y es precisamente el espacio solución el que limita y genera esta oferta de producto, variantes y personalización limitada, en la misma forma que los recursos, capacidades y tecnología disponibles lo son para la empresa. A su vez el espacio solución define el proceso (equipos, tecnología, flexibilidad, sistemas de información, gestión de calidad, etc.) y las operaciones (cadena de suministro, planificación, organización).

Como limitaciones del modelo destacamos, que aunque está basado en una amplia revisión de la literatura y cuenta con la observación del mercado, es un modelo teórico, por ello es 
interesante desarrollar un contraste empírico. Así mismo, al ser un modelo de aplicación general no ha sido posible entrar en las peculiaridades de cada industria. En este sentido se sugiere la adaptación del modelo a diferentes sectores, el desarrollo de cuestionarios que permitan la validación empírica a nivel sectorial así como la utilización de herramientas estadísticas, como por ejemplo ecuaciones estructurales con variables latentes, que permitan analizar el ajuste sectorial del modelo que se plantea.

\section{Referencias}

AHLSTROMP, P.; WESTBROOK, R. (1999). Implications of mass customization for operations management. An exploratory survey. International Journal of Operations \& Production Management, 19(3): 262-275. http://dx.doi.org/10.1108/01443579910249705

AMIT, R; ZOTT, C. (2001). Value creation in e-Business. Strategic Management Journal, 22(6-7): 493-520. http://dx.doi.org/10.1002/smj.187

BLECKER, T.; FRIEDICH, G.; KALUZA, B.; ABDELKAFI, N.; KREUUTLER, G. (2005). Information and Management Systems for Product Customization. Springer's Integrated Series in Information Systems, 5284.

BOHNSTEDT, K.D. (2014). Enabling Facilitation of Mass Customization via Partnering in the Construction Industry. In Proceedings of the 7th World Conference on Mass Customization, Personalization, and Co-Creation (MCPC 2014), Aalborg, Denmark, February 4th-7th, 2014 (pp. 179-188). Springer International Publishing. http://dx.doi.org/10.1007/978-3-319-04271-8_16

CHRISTENSEN, C. (2013). The innovator's dilemma: when new technologies cause great firms to fail. Boston, Cambridge: Harvard Business Review Press.

DA SILVEIRA, G.; BORENSTEIN, D.; FOGLIATTO, F.S. (2001). Mass customization: Literature review and research directions. International Journal of Production Economics, 72(1): 1-13. http://dx.doi.org/10.1016/S0925-5273(00)00079-7

DAVENPORT, T.H.; PRUSAK, L. (1997). Information ecology: Mastering the information and knowledge environment. Oxford: Oxford University Press.

DAMM, R.; DE PABLOS hEREDERO, C.; RODRÍGUEZ MONROY, C. (2013). A Review and a Conceptual Framework of the Key Value Drivers of Mass Customization. International Journal of Technology Marketing, 8(4): 411-430. http://dx.doi.org/10.1504/IJTMKT.2013.056832

DAVIS, S. (1987). Future perfect . Reading: Addison-Wesley. 
DEAN, P.R.; XUE, D.; TU, Y.L. (2009). Prediction of manufacturing resource requirements from customer demands in mass-customisation production. International Journal of Production Research, 47(5): 1245-1268. http://dx.doi.org/10.1080/00207540701557197

DU, X.; JIAO, J.; TSENG, M. (2006). International Journal Adv. Manufacturing Technology, 31: 296-406. http://dx.doi.org/10.1007/s00170-005-0177-8

FIORE, A.M.; LEE, S.E.; KUNZ, G. (2004). Individual differences, motivations, and willingness to use a mass customization option for fashion products. European Journal of Marketing, 38(7): 835-849. http://dx.doi.org/10.1108/03090560410539276

FRANKE, N.; SCHREIER , M. (2008). Product uniqueness as a driver of customer utility in mass customization. Marketing Letters, 19(2): 93-107. http://dx.doi.org/10.1007/s11002-007-9029-7

FRANKE, N.; KEINZ, P.; SCHREIER, M. (2008). Complementing Mass Customization Toolkits with User Communities: How Peer Input Improves Customer Self-Design. Journal of product innovation management, 25(6): 546-559. http://dx.doi.org/10.1111/j.1540-5885.2008.00321.x

FRANKE, N.; VON HIPPEL, E; SCHREIER, M. (2006). Finding Commercially Attractive User Innovations: An Test of Lead User-Theory. Journal of product innovation management, 23(4): 301-315. http://dx.doi.org/10.1111/j.1540-5885.2006.00203.x

GALLEGO GÓMEZ, C.; DE PABLOS HEREDERO, C. (2013). La gamificación y el enriquecimiento de las prácticas de innovación en la empresa: un análisis de experiencias. Revista Intangible Capital, 9(3): 800-822.

GRENCI, R.T.; WATTS, C.A. (2007). Maximizing customer value via mass customized e-consumer services. Business Horizons, 50(2): 123-132.

http://dx.doi.org/10.1016/j.bushor.2006.08.006

HART, C. (1995). Mass customization: Conceptual underpinnings, opportunities and limits. International Journal of Service industry Management, 6(2): 36-45.

http://dx.doi.org/10.1108/09564239510084932

HENARD, D.H.; SZYMANSKI, D.M. (2001). Why some new products are more successful than others. Journal of marketing Research, 38(3): 362-375.

http://dx.doi.org/10.1509/jmkr.38.3.362.18861

JIAO, J.; TSENG, M. (1999). A methodology of developing product family architecture for mass customization. Journal of Intelligence Manufacturing, 10: 3-20.

http://dx.doi.org/10.1023/A:1008926428533

KOTLER, P.; ARMSTRONG, G. (1991). Fundamentos de mercadotecnia. Mexico: Prentice Hall Hispanoamericana. 
KUMAR, A.; GATTOUFI, S.; REISMAN, A. (2007). Mass customization research: trends directions, diffusion intensity, and taxonomic frameworks. International Journal of Flexible Manufacturing Systems, 19: 637-665. http://dx.doi.org/10.1007/s10696-008-9051-y

LAMPEL, J.; MINTZBERG, H. (1996). Customizing Customization. Sloan Management Review, 38(1): 21-30.

LEE, H.; HANG, E. (2011). Consumer attitudes toward online mass customization: an application of extended technology acceptance model'. Journal of Computer-Mediated Communication, 16(2): 171-200. http://dx.doi.org/10.1111/j.1083-6101.2010.01530.x

MACCARTHY, B.; BRABAZON, P.G.; BRAMHAM, J. (2006). Examination of Mass Customization Through Field Evidence. Mass Customization. Challenges and solutions. CIUDAD: Springer.

MOON, H.; LEE, H.H. (2014). Consumers' preference fit and ability to express preferences in the use of online mass customization. Journal of Research in Interactive Marketing, 8(2): 113-124. http://dx.doi.org/10.1108/JRIM-07-2013-0043

PILLER, F. (2005). Mass customization: reflections on the state of the concept. International Journal of Flexible Manufacturing Systems, 16(4): 313-334. http://dx.doi.org/10.1007/s10696005-5170-x

PILLER, F.T.; BLAZEK, P. (2014). Core capabilities of sustainable mass customization. Knowledge based Configuration-From Research to Business Cases (pp. 107-120). Waltham, MA: Morgan Kaufmann Publishers.

PINE, B.J. (1993). Mass customization. Boston: Harvard Business School Press.

PINE, B.J. (1995). Challenges to Total Quality Management in Manufacturing. In James W. Cortada \& J.A. Woods (Eds.), The Quality Yearbook (pp. 69-75). New York: Springer.

PINE, B.J. (1999). Mass customization: the new frontier in business competition. Boston, Cambridge: Harvard Business Press.

PORTER, M.E. (1980). Competitive Strategy: Techniques for Analyzing Industries and Competitors. New York, NY: Free Press.

REICHWALD, R.; PILLER, F.; MOSLEIN, K. (2000). Information as a Critical Success Factor for Mass Customization, Or: Why Even a Customized Shoe Not Always Fits. In Proceedings of the ASAC-IFSAM 2000 Conference.

RICHARDS, K.A.; JONES, E. (2008). Customer relationship management: Finding value drivers. Industrial marketing management, 37(2): 120-130. 
ROBERTS, E.B.; MEYER, M.H. (1991). Product strategy and corporate success. IEEE Engineering management Review, 19(1): 4-18.

SATAM, D.; LIU, Y.; LEE, H.J. (2011). Intelligent design systems for apparel mass customization. The Journal of The Textile Institute, 102(4): 353-365.

http://dx.doi.org/10.1080/00405000.2010.482351

SELLADURAI, R.S. (2004). Mass customization in operations management: oxymoron or reality?. Omega, 32(4): 295-300. http://dx.doi.org/10.1016/j.omega.2003.11.007

SHIMADA, S.; OGATA, T.; OTA, J.; HARA, T. (2014). Constructing Required Functions of Tourism Service Based on Tourists' Expectancy in Trip Planning. Serviceology for Services (pp. 77-83). Japan: Springer.

SPRING, M.; DALRYMPLE, J.F. (2000). Product customization and manufacturing strategy. International Journal of Operations \& Production Management, 20(4): 441-467. http://dx.doi.org/10.1108/01443570010314782

TSENG, M.; PILLER, F. (2003). The Customer Centric Enterprise: Advances in Mass Customization and Personalization. New York/Berlin: Springer. http://dx.doi.org/10.1007/978-3642-55460-5

TSENG, M.M.; JIAO, J. (2001). Mass Customization, Handbook of Industrial Engineering, Technology and Operation Management (pp. 684-709) (3rd ed.). New York, NY: Wiley.

WANG, L.; ZENG, X.; KOEHL, L.; CHEN, Y. (2014). A Human Perception-Based Fashion Design Support System for Mass Customization. Knowledge Engineering and Management (pp. 543-555). Heidelberg: Springer Berlin Heidelberg.

ZHONG, R.Y.; DAI, Q.Y.; QU, T.; HU, G.J.; HUANG, G.Q. (2013). RFID-enabled real-time manufacturing execution system for mass-customization production. Robotics and Computer-Integrated Manufacturing, 29(2): 283-292. http://dx.doi.org/10.1016/j.rcim.2012.08.001

Intangible Capital, 2015 (www.intangiblecapital.org)

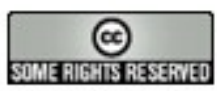

El artículo está con Reconocimiento-NoComercial 3.0 de Creative Commons. Puede copiarlo, distribuirlo y comunicarlo públicamente siempre que cite a su autor y a Intangible Capital. No lo utilice para fines comerciales. La licencia completa se puede consultar en http://creativecommons.org/licenses/by-nc/3.0/es/ 